\title{
Stochastic Epidemic Model of Covid-19 via the Reservoir-People Transmission Network
}

\author{
Kazem Nouri ${ }^{1, *}$, Milad Fahimi ${ }^{1}$, Leila Torkzadeh ${ }^{1}$ and Dumitru Baleanu ${ }^{2,3}$ \\ ${ }^{1}$ Department of Mathematics, Faculty of Mathematics, Statistics and Computer Sciences, Semnan University, P. O. Box \\ 35195-363, Semnan, Iran \\ ${ }^{2}$ Department of Mathematics, Faculty of Arts and Sciences, Cankaya University, Ankara, Turkey \\ ${ }^{3}$ Institute of Space Sciences, Magurele-Bucharest, Romania \\ *Corresponding Author: Kazem Nouri. Email: knouri@semnan.ac.ir; knouri.h@gmail.com \\ Received: 16 October 2021; Accepted: 17 December 2021
}

\begin{abstract}
The novel Coronavirus COVID-19 emerged in Wuhan, China in December 2019. COVID-19 has rapidly spread among human populations and other mammals. The outbreak of COVID-19 has become a global challenge. Mathematical models of epidemiological systems enable studying and predicting the potential spread of disease. Modeling and predicting the evolution of COVID-19 epidemics in near real-time is a scientific challenge, this requires a deep understanding of the dynamics of pandemics and the possibility that the diffusion process can be completely random. In this paper, we develop and analyze a model to simulate the Coronavirus transmission dynamics based on Reservoir-People transmission network. When faced with a potential outbreak, decision-makers need to be able to trust mathematical models for their decision-making processes. One of the most considerable characteristics of COVID-19 is its different behaviors in various countries and regions, or even in different individuals, which can be a sign of uncertain and accidental behavior in the disease outbreak. This trait reflects the existence of the capacity of transmitting perturbations across its domains. We construct a stochastic environment because of parameters random essence and introduce a stochastic version of the Reservoir-People model. Then we prove the uniqueness and existence of the solution on the stochastic model. Moreover, the equilibria of the system are considered. Also, we establish the extinction of the disease under some suitable conditions. Finally, some numerical simulation and comparison are carried out to validate the theoretical results and the possibility of comparability of the stochastic model with the deterministic model.
\end{abstract}

Keywords: Coronavirus; infectious diseases; stochastic modeling; brownian motion; reservoir-people model; transmission simulation; stochastic differential equation 


\section{Introduction}

Mathematical biology is one of the most interesting research areas for applied mathematicians. In these research areas, modeling infectious diseases are considered as an important tool to describe the dynamics of epidemic transmission [1-11]. These models play an important role in quantifying the efficient control and preventive measures of infectious diseases. Different scholars used various types of mathematical models in their analysis.

The ongoing Coronavirus disease 2019 (COVID-19) outbreak, emerged in Wuhan, China at the end of 2019 and increased the attention worldwide [12]. COVID-19 has rapidly spread among human populations and other mammals. Additionally, COVID-19 can be capable of spreading from person to person and between cities. Modeling and predicting the evolution of COVID-19 epidemics in near real-time is a scientific challenge [13], this requires a deep understanding of the dynamics of pandemics and the possibility that the diffusion process can be completely random [14]. Nowadays, a large number of models are used to simulate the current COVID-19 pandemic. One of the common ways for analyzing the dynamic of COVID-19 is the use of ordinary differential equations (ODEs), but there are some limitations compared to stochastic models. Recently, many works related to the ODEs have been published (see, for example [15-19]). Furthermore, Tahir et al. [20] developed a mathematical model (for MERS) in form of a nonlinear system of differential equations. Chen et al. [21] developed a Bats-Hosts-Reservoir-People (BHRP) transmission network model for simulating the phase-based transmissibility of Coronavirus, which focus on calculating $R_{0}$. Additionally, Hashemizadeh et al. [22], presented a numerical solution for the mathematical model of the novel Coronavirus by the application of alternative Legendre polynomials to find the transmissibility of COVID-19 based on ReservoirPeople network model. Also, Gao et al. [23] presented a numerical solution for the BHRP transmission network model for simulating the transmissibility of Coronavirus.

The deterministic models make assumptions about the expected value of parameters in the future, but they ignore the variation and fluctuation about the expected value of parameters. One of the main benefits of the stochastic models is that they allow the validity of assumptions to be tasted statistically and produce an estimate with additional degree of realism. However, there are times when stochastic output cannot be thoroughly reviewed and compared to expectations. Stochastic models have advantages but they are computationally quite complex to perform and need careful consideration of outputs. The parameters of deterministic models governing the equations are supposed to be known and therefore the solutions are often unique. This limitation poses a practical problem because nature is intrinsically heterogeneous and the system is only measured at a discrete (and often small) number of locations. Stochastic models can be regarded as a tool to combine deterministic models, statistics and uncertainty within a coherent theoretical framework. So, this idea leads researchers to consider the stochastic models [3, 24-35]. On the other hand, one of the most considerable characteristics of COVID-19 is its different behaviors in various countries and regions, or even in different individuals, which can be a sign of uncertain and accidental behavior in the disease outbreak. This trait reflects the existence of the capacity of transmitting perturbations across its domains. In past studies, [10-11, 29, 30, 35-39] have been considered a stochastic form of the COVID-19 outbreak.

In this paper, we establish a model for investigation of the COVID-19 transmission based on a stochastic version of the Reservoir-People network with additional degree of realism. Also, it is important to note that an implication of our work is that the numerical simulations demonstrate the efficiency of our model and the possibility of comparability of the stochastic model with the deterministic model. We believe that the stochastic model established by this study is useful for 
researchers and scientists in making informed decisions and taking appropriate steps to dominance the COVID-19 disease. We conduct numerical simulations to support the theoretical findings. Numerical simulations demonstrate the efficiency of our model. We hope that the stochastic model established by this study will be useful for researchers and scientists in making informed decisions and taking appropriate steps to dominance the COVID-19 disease. The mathematical analysis of this stochastic model can be investigated in future researches and the simulation results can be extended to other countries involved in the global outbreak. This paper could lead to other studies that have included random and uncertainty of the model and can be more consistent with the reality of Coronavirus transmission. This paper is organized as follows. In Section 2, preliminaries are presented. In Section 3 , the stochastic version of the Reservoir-People network model of transmission is presented. Section 4 is devoted to present dynamical analysis of solution. Finally, in order to validate our analytical results, numerical simulations are presented in Sections 5 and 6.

\section{Preliminaries}

Let $\left(\Omega,\left\{\mathcal{F}_{t}\right\}_{t \geq 0}, \mathbb{P}\right)$ be a complete probability space with a filtration $\left\{\mathcal{F}_{t}\right\}_{t \geq 0}$ satisfying the usual conditions. We also let $\mathbb{R}_{+}^{d}=\left\{x \in \mathbb{R}^{d}: x_{i}>0,1 \leq i \leq d\right\}, d$-dimensional stochastic differential equation can be expressed as follows:

$d X(t)=f(t, X(t)) d t+g(t, X(t)) d B(t), t \geq t_{0}$,

with initial value $X\left(t_{0}\right)=X_{0}$, where $f(t, x)$ is a function in $\mathbb{R}^{d}$ defined on $\left[t_{0},+\infty\right) \times \mathbb{R}^{d}, g(t, x)$ is a $d \times m$ matrix and $B(t)$ is an $m$-dimensional standard Brownian motion defined on the probability space $\left(\Omega, \mathcal{F}_{t}, \mathbb{P}\right)$, also $f, g$ are locally Lipschitz functions in $x$.

We define the differential operator $L$ associated with Eq. (1) by [39],

$L=\frac{\partial}{\partial t}+\sum_{i=1}^{d} f_{i}(t, x) \frac{\partial}{\partial x_{i}}+\frac{1}{2} \sum_{i, j=1}^{d}\left[g^{T}(t, x) g(t, x)\right]_{i j} \frac{\partial^{2}}{\partial x_{i} \partial x_{j}}$.

If $L$ acts on function $V$ in $C^{2 \times 1}\left(\mathbb{R}^{d} \times\left[t_{0}, \infty\right)\right)$, then we obtain

$L V(t, x)=V_{t}(t, x)+V_{x}(t, x) f(t, x)+\frac{1}{2} \operatorname{trace}\left[g^{T}(t, x) V_{x x}(t, x) g(t, x)\right]$,

where

$V_{t}=\frac{\partial V}{\partial t}, V_{x}=\left(\frac{\partial V}{\partial x_{1} \partial x_{2} \ldots \partial x_{d}}\right)^{T}, V_{x x}=\left(\frac{\partial^{2} V}{\partial x_{i} \partial x_{j}}\right)_{d \times d}$.

The generalized Itô formula implies that

$d V(t, x)=L V(t, x) d t+V_{x}(t, x) g(t, x) d B(t)$.

\section{COVID-19 Model}

In this work, we consider a model of 2019-novel Coronavirus (COVID-19) named as Reservoirpeople model. This model is the simplified and normalized form of Bats-Hosts-Reservoir-People model [21]. Assuming $S_{p}$ and $E_{p}$ refer to the number of susceptible and exposed people, $I_{p}$ denotes symptomatic infected people, $A_{p}$ denotes asymptomatic infected people, $R_{p}$ denotes removed people including recovered and dead people and $W$ denotes the COVID in the reservoir (the seafood market). These variables have the unit of number which are considered at time $t$. Also, the model parameters 
and their definitions are given in Tab. 1. Furthermore, the pictorial diagram indicating of the model is shown in Fig. 1.

Table 1: Model parameters and their definitions

\begin{tabular}{|c|c|}
\hline Parameter & Description \\
\hline$n_{p}$ & The birth rate parameter of people \\
\hline$m_{p}$ & The death rate parameter of people \\
\hline 1 & The incubation period of people \\
\hline$\overline{w_{p}}$ & \\
\hline$\frac{1}{\gamma_{p}^{\prime}}$ & $\begin{array}{l}\text { The infectious period of asymptomatic infection of } \\
\text { people }\end{array}$ \\
\hline$\frac{1}{\gamma_{p}}$ & $\begin{array}{l}\text { The infectious period of symptomatic infection of } \\
\text { people }\end{array}$ \\
\hline$\frac{1}{a,}$ & The latent period of people \\
\hline$w_{p}^{\prime}$ & \\
\hline$\frac{1}{\epsilon}$ & The lifetime of virus in $\mathrm{W}$ \\
\hline$\delta_{p}$ & The proportion of asymptomatic infection of people \\
\hline$\mu_{p}$ & The shedding coefficient from $A_{p}$ to $\mathrm{W}$ \\
\hline$\mu_{p}^{\prime}$ & The shedding coefficient from $I_{p}$ to $\mathrm{W}$ \\
\hline$N_{p}$ & The total number of people \\
\hline$\beta_{p}$ & The transmission rate from $I_{p}$ to $S_{p}$ \\
\hline$\beta_{w}$ & The transmission rate from $W$ to $S_{p}$ \\
\hline
\end{tabular}

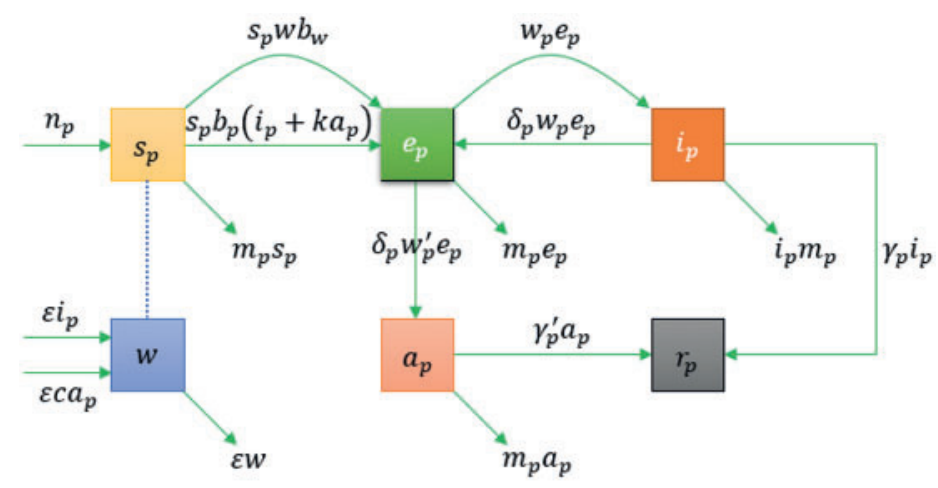

Figure 1: The model's flow diagram

In this paper, we assume $n_{p}$ and $m_{p}$ as the rate of people moving into and moving out from a region per day and the unit of measuring is (individual/day). The incubation period $\left(w_{p}\right)$ is the time between infection or contact with the agent and the onset of symptoms or signs of infection and the unit of measuring is $\left(d a y^{-1}\right)$. In this model, the unit of measuring $w_{p}^{\prime}, \gamma_{p}$ and $\gamma_{p}^{\prime}$ is $\left(d a y^{-1}\right)$. The Coronavirus typically spreads via droplets from an infected person's coughs or sneezes. Coronavirus could survive 
on different types, ranging from $2 \mathrm{~h}$ to 14 days. In this model, the unit of measuring $\epsilon$ is $\left(d a y^{-1}\right)$. Furthermore, the unit of measuring transmission rates $\beta_{p}$ and $\beta_{w}$ is the number of infections per unit time. Also, we defined asymptomatic COVID-19 positive persons as those who did not present any symptoms. The proportion $\delta_{p}$ is calculated as the number of asymptomatic COVID-19 infections at initial testing over COVID-19 positive persons.

The process of normalization is implemented as follows [21]:

$s_{p}=\frac{S_{p}}{N_{p}}, e_{p}=\frac{E_{p}}{N_{p}}, i_{p}=\frac{I_{p}}{N_{p}}$,

$a_{p}=\frac{A_{p}}{N_{p}}, r_{p}=\frac{R_{p}}{N_{p}}, w=\frac{\epsilon W}{\mu_{p} N_{p}}$,

$\mu_{p}^{\prime}=c \mu_{p}, b_{p}=\beta_{p} N_{p}, b_{w}=\frac{\mu_{p} \beta_{w} N_{p}}{\epsilon}$.

Therefore the six-dimensional integer-order COVID-19 model can be expressed by the following ordinary differential equation:

$\dot{s}_{p}=n_{p}-m_{p} s_{p}-s_{p} b_{p}\left(i_{p}+k a_{p}\right)-s_{p} w b_{w}$,

$\dot{e}_{p}=s_{p} b_{p}\left(i_{p}+k a_{p}\right)+b_{w} s_{p} w-w_{p} e_{p}+\delta_{p} w_{p} e_{p}-\delta_{p} w_{p}^{\prime} e_{p}-m_{p} e_{p}$,

$\dot{i}_{p}=w_{p} e_{p}-\delta_{p} w_{p} e_{p}-\gamma_{p} i_{p}-i_{p} m_{p}$,

$\dot{a}_{p}=\delta_{p} w_{p}^{\prime} e_{p}-\gamma_{p}^{\prime} a_{p}-m_{p} a_{p}$,

$\dot{r}_{p}=\gamma_{p} i_{p}+\gamma_{p}^{\prime} a_{p}-r_{p} m_{p}$

$\dot{w}=\epsilon\left(i_{p}+c a_{p}-w\right)$.

\subsection{Implementation of Stochastic Description}

We can provide an additional degree of realism by defining the white noise and Brownian motion and introducing a stochastic model. Therefore, we implement this idea by replacing random parameters

$n_{p} \rightarrow n_{p}+\sigma_{1} \dot{B}_{1}(t), \quad m_{p} \rightarrow m_{p}+\sigma_{2} \dot{B}_{2}(t)$,

$b_{p} \rightarrow b_{p}+\sigma_{3} \dot{B}_{3}(t), \quad b_{w} \rightarrow b_{w}+\sigma_{4} \dot{B}_{4}(t)$,

$\delta_{p} \rightarrow \delta_{p}+\sigma_{5} \dot{B}_{5}(t), \epsilon \rightarrow \epsilon+\sigma_{6} \dot{B}_{6}(t)$,

where $B_{i}(t)$ and $\sigma_{i}, i=1,2, \ldots, 6$ are the Brownian motions and the intensities of the white noises, respectively. These parameters are selected for the implementation of stochastic environment because of their random essence. So, the following stochastic differential equation for COVID-19 model is obtained

$$
\begin{aligned}
s_{p}= & \left(n_{p}-m_{p} s_{p}-s_{p} b_{p}\left(i_{p}+k a_{p}\right)-s_{p} w b_{w}\right) d t+\sigma_{1} d B_{1}-s_{p} \sigma_{2} d B_{2} \\
& -s_{p}\left(i_{p}+k a_{p}\right) \sigma_{3} d B_{3}-s_{p} w \sigma_{4} d B_{4}, \\
e_{p}= & \left(s_{p} b_{p}\left(i_{p}+k a_{p}\right)+b_{w} s_{p} w-w_{p} e_{p}+\delta_{p} w_{p} e_{p}-\delta_{p} w_{p}^{\prime} e_{p}-m_{p} e_{p}\right) d t \\
& +s_{p} \sigma_{3}\left(i_{p}+k a_{p}\right) d B_{3}+s_{p} w \sigma_{4} d B_{4}+w_{p} e_{p} \sigma_{5} d B_{5}-w_{p}^{\prime} e_{p} \sigma_{5} d B_{5}-e_{p} \sigma_{2} d B_{2}, \\
i_{p}= & \left(w_{p} e_{p}-\delta_{p} w_{p} e_{p}-\gamma_{p} i_{p}-i_{p} m_{p}\right) d t-i_{p} \sigma_{2} d B_{2}-\sigma_{5} w_{p} e_{p} d B_{5}, \\
a_{p}= & \left(\delta_{p} w_{p}^{\prime} e_{p}-\gamma_{p}^{\prime} a_{p}-m_{p} a_{p}\right) d t-a_{p} \sigma_{2} d B_{2},
\end{aligned}
$$


$r_{p}=\left(\gamma_{p} i_{p}+\gamma_{p}^{\prime} a_{p}-r_{p} m_{p}\right) d t-r_{p} \sigma_{2} d B_{2}$,

$w=\epsilon\left(i_{p}+c a_{p}-w\right) d t+\sigma_{6}\left(i_{p}+c a_{p}-w\right) d B_{6}$.

\section{Dynamical Analysis of Solution}

In this section, existence and uniqueness of the solution of the introduced model (2) and the equilibria of the system are presented.

\subsection{Existence and Uniqueness of the Solution}

Theorem 4.1. The coefficients of the stochastic differential Eq. (2) are locally Lipschitz.

Proof. We define the following kernel $\gamma\left(s_{p}, i_{p}\right)=s_{p} . i_{p}$. Now we should prove the Lipschitz condition for $\gamma\left(s_{p}, i_{p}\right)$. First we set $\mathrm{d}=\left(s_{p}, i_{p}\right)$ therefore $\gamma(\mathrm{d})=s_{p}, i_{p}$ and $\gamma(d) \in C^{1}(\Omega, \mathrm{R})$. So we must prove $\forall d_{1}, d_{2} \in \Omega, \exists K>0:\left\|\gamma\left(d_{2}\right)-\gamma\left(d_{1}\right)\right\| \leq K\left\|d_{2}-d_{1}\right\|$

Now we choose suitable a subset of $\Omega$ and consider $N(x, r)$ as an open ball centered at $x$ and with radius $r$, such that

$N(x, r)=\{y \mid\|y-x\| \leq r\}$

Then we continue our proof by applying: $d_{1}, d_{2} \in N(x, r) \subset \Omega \subset \mathbb{R}^{2}$. Since $\gamma(\mathrm{d})$ is unbounded therefore, due to (3), we replace $\mathrm{K}$ with $K_{x}$ which depends on $x$.

So we define the path $\psi:[0,1] \rightarrow N(x, r)$ such that $\psi(t)=(1-t) d_{1}+t d_{2}$. So. We consider the following increment from $\mathrm{d}_{1}$ to $\mathrm{d}_{2}$ :

$\left\|\gamma\left(d_{2}\right)-\gamma\left(d_{1}\right)\right\|=\|\gamma(\psi(1))-\gamma(\psi(0))\|=\int_{0}^{1} \frac{d \gamma(\psi(t))}{d t} d t$

where $\frac{d \gamma(\psi(t))}{d t}=\nabla \gamma(\psi(t)) \psi^{\prime}(t)=\nabla \gamma(\psi(t))\left(d_{2}-d_{1}\right)$. Thus we have:

$\left\|\int_{0}^{1} \frac{d \gamma(\psi(t))}{d t} d t\right\|=\left\|\int_{0}^{1} \nabla \gamma(\psi(t))\left(d_{2}-d_{1}\right) d t\right\| \leq \int_{0}^{1}\|\nabla \gamma(\psi(t))\|\left\|d_{2}-d_{1}\right\| d t$

$=\left\|d_{2}-d_{1}\right\| \int_{0}^{1}\|\nabla \gamma(\psi(t))\| d t$

$\leq K_{x}\left\|d_{2}-d_{1}\right\|$

Where $K_{x}>\sup \{\|\nabla \Upsilon(d) \mid d \in N(x, r)\|\}$.

As a result, there is no global Lipschitz constant $\mathrm{K}$ valid on all of $\Omega=\mathbb{R}^{2}$ in this case, thus $\gamma$ (d) is in fact locally Lipschitz.

Lemma 4.1. Suppose that $X \subset R_{n}$, and let f, g: $X \subset R_{n}$ satisfy Lipschitz conditions, then $f+g$ satisfies Lipschitz condition.

Proof. The proof is presented in [40].

Therefore, we have local Lipschitz condition for the coefficient of $d B_{1}(t)$ and similarly, this condition will be proved for the coefficient of $d B_{2}(t), d B_{3}(t), d B_{4}(t), d B_{5}(t)$ and $d B_{6}(t)$. So our Model contains stochastic differential equations with Lipschitz coefficient and we can obtain a strong solution and for each initial value, the solution will be unique. 
Theorem 4.2. For any given initial value $\left(s_{p}(0), e_{p}(0), i_{p}(0), a_{p}(0), r_{p}(0), w(0)\right) \in R^{6}$, there exists the solution $\left(s_{p}(\mathrm{t}), e_{p}(\mathrm{t}), i_{p}(\mathrm{t}), a_{p}(\mathrm{t}), r_{p}(\mathrm{t}), \mathrm{w}(\mathrm{t})\right)$ for model $(2)$ and the solution will remain in $R^{6}{ }_{+}$with probability one.

Proof. There exists a local solution on the interval $\left[0, \tau_{e}\right)$ where $\tau_{e}$ is the explosion time [39]. Now, we need to prove $\tau_{e}=+\infty$. Let $m_{0} \geq 0$ be sufficiently large such that each component of initial value $\left(s_{p}(0), e_{p}(0), i_{p}(0), a_{p}(0), r_{p}(0), \mathrm{w}(0)\right)$ remain within the interval $\left[\frac{1}{m_{0}}, m_{0}\right]$. For each integer $m \geq m_{0}$, we define

$\tau_{m}=\inf \left\{t \in\left[0, \tau_{e}\right): \min \left\{\left(s_{p}(t), e_{p}(t), i_{p}(t), a_{p}(t), r_{p}(t), w(t)\right)\right\} \leq \frac{1}{m}\right\}$

or

$\tau_{m}=\inf \left\{t \in\left[0, \tau_{e}\right): \max \left\{\left(s_{p}(t), e_{p}(t), i_{p}(t), a_{p}(t), r_{p}(t), w(t)\right)\right\} \geq m\right\}$

where $\tau_{\mathrm{m}}$ is stopping time. We denote $\tau_{+\infty}=\lim _{\mathrm{m} \rightarrow \infty} \tau_{\mathrm{m}}$ and we will show that $\tau_{+\infty}=+\infty$ is valid almost surely. Then the proof goes by contradiction. If this statement is false, then there exist constants $\mathrm{T} \geq 0$ and $\eta \in(0,1)$ such that $\mathrm{P}\left\{\tau_{\mathrm{m}} \leq \mathrm{T}\right\} \geq \eta$ for any $\mathrm{m} \geq \mathrm{m}_{0}$.

Let $\mathrm{f}$ be a $C^{2}$-function, by defining $f: \mathbb{R}_{+}^{6} \rightarrow \mathbb{R}$ such that $f\left(s_{p}(t), e_{p}(t), i_{p}(t), a_{p}(t), r_{p}(t), w(t)\right)=\ln s_{p}(t)+\ln e_{p}(t)+\ln i_{p}(t)+\ln a_{p}(t)+\ln r_{p}(t)+\ln w(t)$

Generalized Itô's formula yields that

$$
\begin{aligned}
d f\left(t, s_{p}, e_{p}, i_{p}, a_{p}, w\right)= & f_{s_{p}} d s_{p}+f_{e_{p}} d_{e_{p}}+f_{i_{p}} d_{i_{p}}+f_{a_{p}} d_{a_{p}}+f_{r_{p}} d_{r_{p}}+f_{w} d_{w}+ \\
& +\frac{1}{2}\left(f_{s_{p} s_{p}} d_{s_{p}} d_{s_{p}}+f_{e_{p} e_{p}} d_{e_{p}} d_{e_{p}}+f_{i_{p} i_{p}} d_{i_{p}} d_{i_{p}}\right. \\
& \left.+f_{a_{p} a_{p}} d_{a_{p}} d_{a_{p}}+f_{r_{p} r_{p}} d_{r_{p}} d_{r_{p}}+f_{w w} d_{w} d_{w}\right)+\ldots
\end{aligned}
$$

So we obtain

$$
\begin{aligned}
d_{f}= & \frac{n_{p}}{n_{s}}-5 m_{p}-b_{p}\left(i_{p}+k \cdot a_{p}\right)-w \cdot b_{w}+\frac{s_{p} b_{p}}{e_{p}}\left(i_{p}+k a_{p}\right)+\frac{b_{w} \cdot s_{p} \cdot w}{e_{p}}-w_{p}+\delta_{p} w_{p}-\delta_{p} w_{p}^{\prime} \\
& +\frac{w_{p} e_{p}}{i_{p}}-\frac{\sigma_{p} w_{p} e_{p}}{i_{p}}-\gamma_{p}+\frac{\delta_{p} w_{p}^{\prime} e_{p}}{a_{p}}-\gamma_{p}^{\prime}+\frac{\gamma_{p} i_{p}}{r_{p}}+\frac{\gamma_{p}^{\prime} a_{p}}{r_{p}}+\varepsilon\left(\frac{i_{p}+C \cdot a_{p}}{w}-1\right) \\
& -\frac{1}{2}\left(\frac{\sigma_{1}^{2}}{s_{p}^{2}}+5 \sigma_{2}^{2}+\sigma_{3}^{2}\left(i_{p}+k \cdot a_{p}\right)^{2}+w^{2} \sigma_{4}^{2}+\frac{w^{2} \sigma_{4}^{2} s_{p}^{2}}{e_{p}^{2}}+w_{p}^{2} \sigma_{5}^{2}+w_{p}^{\prime} \sigma_{5}^{2}+\frac{\sigma_{5}^{2} w_{p}^{2} e_{p}^{2}}{i_{p}^{2}}\right. \\
& \left.+\frac{\sigma_{6}^{2}\left(i_{p}+C \cdot a_{p}-w\right)^{2}}{w^{2}}+\frac{s_{p}^{2} \sigma_{3}^{2}\left(i_{p}+k \cdot a_{p}\right)^{2}}{e_{p}^{2}}\right)+\frac{\sigma_{1}}{s_{p}} d B_{1}-5 \sigma_{2} d B_{2}+\left(\frac{s_{p}}{e_{p}}-1\right)\left(i_{p}+K a_{p}\right) \sigma_{3} d B_{3} \\
& +w \sigma_{4}\left(\frac{s_{p}}{e_{p}}-1\right) d B_{4}+\left(w_{p}\left(1-\frac{e_{p}}{i_{p}}\right)-w_{p}^{\prime}\right) \sigma_{5} d B_{5}+\sigma_{6} \varepsilon\left(\frac{i_{p}+C \cdot a_{p}}{w}-1\right) d B_{6}
\end{aligned}
$$


Therefore we obtain

$$
\begin{aligned}
d f=L f d t & +\frac{\sigma_{1}}{s_{p}} d B_{1}-5 \sigma_{2} d B_{2}+\left(\frac{s_{p}}{e_{p}}-1\right)\left(i_{p}+K a_{p}\right) \sigma_{3} d B_{3}+w \sigma_{4}\left(\frac{s_{p}}{e_{p}}-1\right) d B_{4} \\
& +\left(w_{p}\left(1-\frac{e_{p}}{i_{p}}\right)-w_{p}^{\prime}\right) \sigma_{5} d B_{5}+\sigma_{6} \varepsilon\left(\frac{i_{p}+C \cdot a_{p}}{w}-1\right) d B_{6}
\end{aligned}
$$

where $\mathrm{K}$ is a positive constant and we have

$$
\begin{aligned}
L f & =\frac{n_{p}}{n_{s}}-5 m_{p}-b_{p}\left(i_{p}+k \cdot a_{p}\right)-w \cdot b_{w}+\frac{s_{p} b_{p}}{e_{p}}\left(i_{p}+k \cdot a_{p}\right)+\frac{b_{w} \cdot s_{p} \cdot w}{e_{p}} \\
& -w_{p}+\sigma_{p} w_{p}-\sigma_{p} w_{p}^{\prime}+\frac{w_{p} e_{p}}{i_{p}}-\frac{\delta_{p} w_{p} e_{p}}{i_{p}}-\gamma_{p}+\frac{\delta_{p} w_{p}^{\prime} e_{p}}{a_{p}}-\gamma_{p}^{\prime}+\frac{\gamma_{p} i_{p}}{r_{p}} \\
& +\frac{\gamma_{p}^{\prime} a_{p}}{r_{p}}+\varepsilon\left(\frac{i_{p}+C \cdot a_{p}}{w}-1\right)-\frac{1}{2}\left(\frac{\sigma_{1}^{2}}{s_{p}^{2}}+5 \sigma_{2}^{2}+\sigma_{3}^{2}\left(i_{p}+k \cdot a_{p}\right)^{2}+w^{2} \sigma_{4}^{2}\right. \\
& +\frac{w^{2} \sigma_{4}^{2} s_{p}^{2}}{e_{p}^{2}}+w_{p}^{2} \sigma_{5}^{2}+w_{p}^{\prime} \sigma_{5}^{2}+\frac{\sigma_{5}^{2} w_{p}^{2} e_{p}^{2}}{i_{p}^{2}}+\frac{\sigma_{6}^{2}\left(i_{p}+C \cdot a_{p}-w\right)^{2}}{w^{2}} \\
& \left.+\frac{s_{p}^{2} \sigma_{3}^{2}\left(i_{p}+k \cdot a_{p}\right)^{2}}{e_{p}^{2}}\right) \leq \frac{n_{p}}{n_{s}}+\frac{s_{p} b_{p}}{e_{p}}\left(i_{p}+K \cdot a_{p}\right)+\frac{b_{w} \cdot s_{p} \cdot w}{e_{p}}+\delta_{p} w_{p} \\
& +\frac{w_{p} e_{p}}{i_{p}}+\frac{\delta_{p} w_{p}^{\prime} e_{p}}{a_{p}}+\frac{\gamma_{p} i_{p}}{r_{p}}+\frac{\gamma_{p}^{\prime} a_{p}}{r_{p}}+\varepsilon\left(\frac{i_{p}+C \cdot a_{p}}{w}-1\right) \leq K
\end{aligned}
$$

Due to (7) and integrating both sides of (6) from to $\left(\tau_{m} \wedge T\right)$ and taking expectation deduce that $E f \leq f\left(s_{p}(0), e_{p}(0), i_{p}(0), a_{p}(0), r_{p}(0), w(0)\right)+K T$

Thus for every $\omega \in \Omega_{m}=\left\{\tau_{m} \leq T\right\}$ we obtain

$$
\begin{aligned}
& f\left(s_{p}(0), e_{p}(0), i_{p}(0), a_{p}(0), r_{p}(0), w(0)\right)+K T \geq E\left[1_{\Omega_{m}}(w) . f\left(s\left(\tau_{m} \wedge T\right), e_{p}\left(\tau_{m} \wedge T\right), i_{p}\left(\tau_{m} \wedge T\right)\right.\right. \\
& \left.\left., a_{p}\left(\tau_{m} \wedge T\right), r_{p}\left(\tau_{m} \wedge T\right), w\left(\tau_{m} \wedge T\right)\right)\right] \\
& \geq p\left\{\tau_{m} \leq T\right\} \cdot[(\ln m) \wedge(-\ln m)] \\
& \geq \eta[(\ln m) \wedge(-\ln m)]
\end{aligned}
$$

where $1_{\Omega_{m}(\omega)}$ is indicator function of $\Omega$. Letting $m \rightarrow+\infty$ leads to contradiction $+\infty>f\left(s_{p}(0), e_{p}(0), i_{p}(0), a_{p}(0), r_{p}(0), w(0)\right)+K T \geq+\infty$.

So the statement $\tau_{+\infty}=+\infty$ is being satisfied and the proof is complete.

\subsection{Extinction of the Disease}

In this section, we establish sufficient conditions for the extinction of the disease. In order to form these conditions, we consider variation in the number of symptomatic infected people $\left(I_{p}\right)$. Chen et al. 
[21] have proved that the deterministic model admits the basic reproduction number $R_{0}$ as follows:

$$
\begin{aligned}
& R_{0}=b_{p} \psi+\kappa b_{p} \psi^{\prime}+b_{w} \psi+b_{w} c \psi^{\prime} \\
& \text { where } \\
& \psi=b_{p} \frac{n_{p}}{m_{p}} \frac{\left(1-\delta_{p}\right) w_{P}}{\left(1-\delta_{p}\right) w_{p}+\delta_{p} w_{p}^{\prime}+m_{p}\left(\gamma_{p}+m_{p}\right)} \text { and } \\
& \psi^{\prime}=\kappa b_{p} \frac{n_{p}}{m_{p} w_{p}^{\prime}} \frac{\left.\delta_{p}\right) w_{P}+\delta_{p} w_{p}^{\prime}+m_{p}\left(\gamma_{p}^{\prime}+m_{p}\right)}{\left(1-\delta_{p}\right.}
\end{aligned}
$$

In deterministic epidemiological models, basic reproduction number is planned to be a criterion of transmissibility of infectious agents. If $R_{0}>1$, then the outbreak is expected to continue if $R_{0}<1$ then the outbreak is expected to become extinct. In this stochastic COVID-19 model, we consider the effect of high intensity white noises on the prevalence or extinction of the COVID-19. So we present the following theorem to demonstrate that random effect may lead the disease to extinct under suitable conditions, although the disease may be immanent for the deterministic model. Before presenting the main theorem of this section, we refer to the following lemma presented in [39].

Lemma 4.2. Let $\mathrm{Z}(\mathrm{t}): \mathrm{t} \geq 0$ be a continuous local martingale [41] and $<\mathrm{Z}(\mathrm{t}), \mathrm{Z}(\mathrm{t})>$ be its quadratic variation. Let $0<\mathrm{c}<1$ and $\mathrm{k}$ be a random integer. Then for almost all $\omega \in \Omega$, there exit a random integer $\mathrm{k}_{0}(\omega)$ such that for all $\mathrm{k}>\mathrm{k}_{0}$ :

$\mathrm{P}\left\{\sup _{0 \leq \mathrm{t} \leq \mathrm{k}}\left[\mathrm{Z}(\mathrm{t})-\frac{\mathrm{c}}{2}<\mathrm{Z}(\mathrm{t}), \mathrm{Z}(\mathrm{t})>\right] \geq \frac{2}{\mathrm{c}} \ln \mathrm{k}\right\} \leq \frac{1}{\frac{2}{\mathrm{c}}}$

Theorem 4.3. Let $\left(s_{p}(0), e_{p}(0), i_{p}(0), a_{p}(0), r_{p}(0), w(0)\right) \in R^{6}{ }_{+}$be the solution of model (2) with initial value $\left(s_{p}(0), \quad e_{p}(0), \quad i_{p}(0), \quad a_{p}(0), r_{p}(0), w(0)\right) \in R^{6}{ }_{+}$, we obtain

$\lim _{\mathrm{t} \rightarrow+\infty} \sup \frac{\ln \mathrm{I}(\mathrm{t})}{\mathrm{t}} \leq \frac{1}{2 \sigma_{5}^{2}}-\left(\frac{1}{2} \sigma_{2}^{2}+\Upsilon_{\mathrm{p}}+\mathrm{m}_{\mathrm{p}}\right)$ a.s.

Furthermore, if the assumptions $\frac{1}{2 \sigma_{5}^{2}} \leq \frac{1}{2 \sigma_{2}^{2}}+\Upsilon_{\mathrm{p}}+\mathrm{m}_{\mathrm{p}}$ and $\delta_{\mathrm{p}}>1$ hold, then $\mathrm{I}(\mathrm{t})$ will tend to zero exponentially with probability one.

Proof. Itô's formula yields that

$\mathrm{d}\left(\ln \mathrm{i}_{\mathrm{p}}\right)=\left(\frac{\omega_{\mathrm{p}} \mathrm{e}_{\mathrm{p}}}{\mathrm{i}_{\mathrm{p}}}-\frac{\delta_{\mathrm{p}} \omega_{\mathrm{p}} \mathrm{e}_{\mathrm{p}}}{\mathrm{i}_{\mathrm{p}}}-\Upsilon_{\mathrm{p}}-\mathrm{m}_{\mathrm{p}}\right) \mathrm{dt}-\sigma_{2} \mathrm{~dB}_{2}(\mathrm{t})-\frac{\sigma_{5} \omega_{\mathrm{p}} \mathrm{e}_{\mathrm{p}}}{\mathrm{i}_{\mathrm{p}}} d B_{5}(t)$

So we obtain

$\ln \left(\mathrm{i}_{\mathrm{p}}\right)=\ln \left(\mathrm{i}_{\mathrm{p}}(0)\right)+\int_{0}^{\mathrm{t}}\left(\frac{\omega_{\mathrm{p}} \mathrm{e}_{\mathrm{p}}}{\mathrm{i}_{\mathrm{p}}}-\frac{\delta_{\mathrm{p}} \omega_{\mathrm{p}} \mathrm{e}_{\mathrm{p}}}{\mathrm{i}_{\mathrm{p}}}-\Upsilon_{\mathrm{p}}-\mathrm{m}_{\mathrm{p}}-\frac{1}{2} \sigma_{2}^{2}-\frac{1}{2} \frac{\sigma_{5}^{2} \omega_{\mathrm{p}}^{2} \mathrm{e}_{\mathrm{p}}^{2}}{\mathrm{i}_{\mathrm{p}}^{2}}\right) \mathrm{dt}-\sigma_{2} \mathrm{~B}_{2}(\mathrm{t})+\mathrm{Z}(\mathrm{t})$.

where $\mathrm{Z}(\mathrm{t})=-\int \frac{\sigma_{5} \mathrm{p}_{\mathrm{p}} \mathrm{p}}{\mathrm{i}_{\mathrm{p}}} \mathrm{dB}_{5}(\mathrm{t})$ is a continuous local martingale and its quadratic variation is

$<\mathrm{Z}(\mathrm{t}), \mathrm{Z}(\mathrm{t})>=\sigma_{5}^{2} \int \frac{\omega_{\mathrm{p}}^{2} \mathrm{e}_{\mathrm{p}}^{2}}{\mathrm{i}_{\mathrm{p}}^{2}} \mathrm{dt}$ 
According to Lemma 4.2, we obtain

$\mathrm{P}\left\{\sup _{0 \leq \mathrm{t} \leq \mathrm{k}}\left[\left(\mathrm{Z}(\mathrm{t})-\frac{\mathrm{c}}{2}\langle\mathrm{Z}(\mathrm{t}), \mathrm{Z}(\mathrm{t})\rangle\right) \geq \frac{2}{\mathrm{c}} \ln \mathrm{k}\right]\right\} \leq \frac{1}{\frac{2}{\mathrm{c}}}$

where $0<\mathrm{c}<1$ and $\mathrm{k}$ is a random integer. Applying Borel-Cantelli Lemma [41] leads to that for almost all $\omega \in \Omega$, there exit a random integer $\mathrm{k}_{0}(\omega)$ such that for $\mathrm{k}>\mathrm{k}_{0}$ :

$\sup _{0 \leq t \leq k}\left[\left(Z(t)-\frac{c}{2}\langle Z(t), Z(t)\rangle\right] \leq \frac{2}{c} \ln k\right.$

Therefore

$\int_{0}^{\mathrm{t}} \frac{\sigma_{5} \omega_{\mathrm{p}} \mathrm{e}_{\mathrm{p}}}{\mathrm{i}_{\mathrm{p}}} \mathrm{dB}_{5}(\mathrm{t}) \leq \frac{1}{2} \sigma_{5}^{2} \int_{0}^{\mathrm{t}} \frac{\omega_{\mathrm{p}}^{2} \mathrm{e}_{\mathrm{p}}^{2}}{\mathrm{i}_{\mathrm{p}}^{2}} \mathrm{dt}+\frac{2}{\mathrm{c}} \ln \mathrm{k}$

for $0 \leq \mathrm{t} \leq \mathrm{k}$. Substituting (9) into (8) yields

$$
\begin{aligned}
\ln \mathrm{I}(\mathrm{t}) \leq \ln \mathrm{i}_{\mathrm{p}}(0) & +\int_{0}^{\mathrm{t}}\left(\frac{\omega_{\mathrm{p}} \mathrm{e}_{\mathrm{p}}}{\mathrm{i}_{\mathrm{p}}}-\frac{\delta_{\mathrm{p}} \omega_{\mathrm{p}} \mathrm{e}_{\mathrm{p}}}{\mathrm{i}_{\mathrm{p}}}-\Upsilon_{\mathrm{p}}-\mathrm{m}_{\mathrm{p}}\right) \mathrm{dt}+\sigma_{2} \mathrm{~B}_{2}(\mathrm{t})+\frac{1}{2} \mathrm{c} \sigma_{5}^{2} \int \frac{\omega_{\mathrm{p}}^{2} \mathrm{e}_{\mathrm{p}}^{2}}{\mathrm{i}_{\mathrm{p}}^{2}} \mathrm{dt}+\frac{2}{\mathrm{c}} \ln \mathrm{k}-\frac{1}{2} \delta_{2}^{2} \\
& -\frac{1}{2} \frac{\sigma_{5}^{2} \omega_{\mathrm{p}}^{2} \mathrm{e}_{\mathrm{p}}^{2}}{\mathrm{i}_{\mathrm{p}}^{2}}=\int_{0}^{\mathrm{t}}\left(\frac{\omega_{\mathrm{p}} \mathrm{e}_{\mathrm{p}}}{\mathrm{i}_{\mathrm{p}}}-\frac{\delta_{\mathrm{p}} \omega_{\mathrm{p}} \mathrm{e}_{\mathrm{p}}}{\mathrm{i}_{\mathrm{p}}}-\Upsilon_{\mathrm{p}}-\mathrm{m}_{\mathrm{p}}-\frac{1}{2}\left(\delta_{2}^{2}+\frac{\delta_{5}^{2} \omega_{\mathrm{p}}^{2} \mathrm{e}_{\mathrm{p}}^{2}}{\mathrm{i}_{\mathrm{p}}^{2}}\right)+\frac{1}{2} \frac{\mathrm{c} \delta_{5}^{2} \omega_{\mathrm{p}}^{2} \mathrm{e}_{\mathrm{p}}^{2}}{\mathrm{i}_{\mathrm{p}}^{2}}\right) \mathrm{dt} \\
& +\delta_{2} \mathrm{~B}_{2}(\mathrm{t})+\frac{2}{\mathrm{c}} \ln \mathrm{k}=\int_{0}^{\mathrm{t}}\left(\frac{\omega_{\mathrm{p}} \mathrm{e}_{\mathrm{p}}}{\mathrm{i}_{\mathrm{p}}}-\frac{\delta_{\mathrm{p}} \omega_{\mathrm{p}} \mathrm{e}_{\mathrm{p}}}{\mathrm{i}_{\mathrm{p}}}-\Upsilon_{\mathrm{p}}-\mathrm{m}_{\mathrm{p}}-\frac{1}{2} \sigma_{2}^{2}-\frac{1}{2}(1-\mathrm{c}) \frac{\sigma_{5}^{2} \omega_{\mathrm{p}}^{2} \mathrm{e}_{\mathrm{p}}^{2}}{\mathrm{i}_{\mathrm{p}}^{2}}\right) \mathrm{dt} \\
& +\sigma_{2} \mathrm{~B}_{2}(\mathrm{t})+\frac{2}{\mathrm{c}} \ln \mathrm{k} .
\end{aligned}
$$

If $\delta_{\mathrm{p}}>1$, we obtain

$$
\begin{aligned}
& \frac{\omega_{\mathrm{p}} \mathrm{e}_{\mathrm{p}}}{\mathrm{i}_{\mathrm{p}}}-\frac{\delta_{\mathrm{p}} \omega_{\mathrm{p}} \mathrm{e}_{\mathrm{p}}}{\mathrm{i}_{\mathrm{p}}}-\frac{1}{2}(1-\mathrm{c}) \frac{\sigma_{5}^{2} \omega_{\mathrm{p}}^{2} \mathrm{e}_{\mathrm{p}}^{2}}{\mathrm{i}_{\mathrm{p}}^{2}}=\left(1-\delta_{\mathrm{p}}\right) \frac{\omega_{\mathrm{p}} \mathrm{e}_{\mathrm{p}}}{\mathrm{i}_{\mathrm{p}}}-\frac{1}{2}(1-\mathrm{c}) \frac{\sigma_{2}^{2} \omega_{\mathrm{p}}^{2} \mathrm{e}_{\mathrm{p}}^{2}}{\mathrm{i}_{\mathrm{p}}^{2}} \\
& \leq-\frac{\sigma_{5}^{2}}{2}(1-\mathrm{c})\left(\frac{-2 \omega_{\mathrm{p}} \mathrm{e}_{\mathrm{p}}}{(1-\mathrm{c}) \mathrm{i}_{\mathrm{p}} \sigma_{5}^{2}}+\frac{\omega_{\mathrm{p}}^{2} \mathrm{e}_{\mathrm{p}}^{2}}{\mathrm{i}_{\mathrm{p}}^{2}}\right) \\
& =-\frac{\sigma_{2}^{2}}{2}(1-\mathrm{c})\left(\frac{\omega_{\mathrm{p}}^{2} \mathrm{e}_{\mathrm{p}}^{2}}{\mathrm{i}_{\mathrm{p}}^{2}}-\left(\frac{2 \omega_{\mathrm{p}} \mathrm{e}_{\mathrm{p}}}{(1-\mathrm{c}) \mathrm{i}_{\mathrm{p}} \sigma_{5}^{2}}+\frac{1}{\sigma_{5}^{4}(1-\mathrm{c})^{2}}-\frac{1}{\sigma_{5}^{4}(1-\mathrm{c})^{2}}\right)\right. \\
& =-\frac{\sigma_{5}^{2}}{2}(1-\mathrm{c})\left(\frac{\omega_{p} e_{p}}{i_{p}}-\frac{1}{\sigma_{5}^{2}(1-c)}\right)^{2}+\frac{1}{2 \sigma_{5}^{2}(1-\mathrm{c})} \leq \frac{1}{2 \sigma_{5}^{2}(1-\mathrm{c})}
\end{aligned}
$$

So we have

$$
\begin{aligned}
& \ln \mathrm{I}(\mathrm{t}) \leq \ln \mathrm{I}(\mathrm{o})+\int_{0}^{\mathrm{t}}\left(-\frac{1}{2} \sigma_{2}^{2}-\Upsilon_{\mathrm{p}}-\mathrm{m}_{\mathrm{p}}+\frac{1}{2 \sigma_{5}^{2}(1-\mathrm{c})}\right) \mathrm{dt}+\sigma_{2} \mathrm{~B}_{2}(\mathrm{t})+\frac{2}{\mathrm{c}} \ln \mathrm{k} \\
& =\ln \mathrm{I}(0)+\left(-\frac{1}{2} \sigma_{2}^{2}-\Upsilon_{\mathrm{p}}-\mathrm{m}_{\mathrm{p}}+\frac{1}{2 \sigma_{5}^{2}(1-\mathrm{c})}\right) \mathrm{t}+\sigma_{2} \mathrm{~B}_{2}(\mathrm{t})+\frac{2}{\mathrm{c}} \ln \mathrm{k} .
\end{aligned}
$$

By dividing both sides of Eq. (10) by $\mathrm{t}$ such that $\mathrm{k}-1 \leq \mathrm{t} \leq \mathrm{k}$, we achive $\frac{\ln \mathrm{I}(\mathrm{t})}{\mathrm{t}} \leq \frac{\ln \mathrm{I}(0)}{\mathrm{t}}+\left(-\frac{1}{2} \sigma_{2}^{2}-\Upsilon_{\mathrm{p}}-\mathrm{m}_{\mathrm{p}}+\frac{1}{2 \sigma_{5}^{2}(1-\mathrm{c})}\right)+\frac{\sigma_{2} \mathrm{~B}_{2}(\mathrm{t})}{\mathrm{t}}+\frac{2}{\mathrm{ct}} \ln \mathrm{k}$. 
Letting $\mathrm{k} \rightarrow \infty$ leads to $\mathrm{n} \rightarrow \infty$ and according to the strong law of large numbers [41] to the Brownian motion, we have $\lim \sup _{t \rightarrow+\infty} \frac{B_{2}(t)}{t}=0$

So we obtain

$\lim _{\mathrm{t} \rightarrow+\infty} \sup \frac{\ln \mathrm{I}(\mathrm{t})}{\mathrm{t}} \leq-\left(\frac{1}{2} \sigma_{2}^{2}+\Upsilon_{\mathrm{p}}+\mathrm{m}_{\mathrm{p}}\right)+\frac{1}{2 \sigma_{5}^{2}(1-\mathrm{c})}$

Letting $\mathrm{c} \rightarrow 0$ yields

$\lim _{\mathrm{t} \rightarrow+\infty} \sup \frac{\ln \mathrm{I}(\mathrm{t})}{\mathrm{t}} \leq \frac{1}{2 \sigma_{5}^{2}}-\left(\frac{1}{2} \sigma_{2}^{2}+\Upsilon_{\mathrm{p}}+\mathrm{m}_{\mathrm{p}}\right)$

Therefore, our claim holds and this completes our proof.

Remark 4.1. Theorem 4.3 shows that the disease will decay exponentially to zero under suitable conditions. When the noises $\sigma_{2}$ and $\sigma_{5}$ are sufficiently large then the disease goes extinct exponentially. The white noises $\sigma_{2}$ and $\sigma_{5}$ are related to the fluctuation of $m_{p}$ (the death rate of people) and $\delta_{p}$ (the proportion of asymtomatic infection of people), respectively.

\subsection{Equilibria of the System}

Equilibria of the system can be estimated by setting the all derivatives equal to zero. This gives the system

$n_{p}-m_{p} s_{p}-s_{p} b_{p}\left(i_{p}+k a_{p}\right)-s_{p} w b_{w}=0$,

$s_{p} b_{p}\left(i_{p}+k a_{p}\right)+b_{w} s_{p} w-w_{p} e_{p}+\delta_{p} w_{p} e_{p}-\delta_{p} w_{p}^{\prime} e_{p}-m_{p} e_{p}=0$,

$\left(w_{p} e_{p}-\delta_{p} w_{p} e_{p}-\gamma_{p} i_{p}-i_{p} m_{p}\right) d t-i_{p} \sigma_{2} d B_{2}-\sigma_{5} w_{p} e_{p} d B_{5}=0$,

$\delta_{p} w_{p}^{\prime} e_{p}-\gamma_{p}^{\prime} a_{p}-m_{p} a_{p}=0$,

$\gamma_{p} i_{p}+\gamma_{p}^{\prime} a_{p}-r_{p} m_{p}=0$,

$\epsilon\left(i_{p}+c a_{p}-w\right)=0$.

In order to obtain the fixed points of COVID-19 model (2), we consider the solution of system (11) as follows

$\dot{\mathrm{s}}_{\mathrm{p}}=0$, we obtain $\tilde{\mathrm{s}}_{\mathrm{p}}=\frac{\mathrm{n}_{\mathrm{p}}}{\mathrm{m}_{\mathrm{p}}-\mathrm{b}_{\mathrm{p}}\left(\mathrm{i}_{\mathrm{p}}+\mathrm{ka}_{\mathrm{p}}\right)-\mathrm{wb}_{\mathrm{w}}}$.

For $\dot{\mathrm{e}}_{\mathrm{p}}=0$, we obtain $\tilde{\mathrm{e}}_{\mathrm{p}}=\frac{\mathrm{s}_{\mathrm{p}} \mathrm{b}_{\mathrm{p}}\left(\mathrm{i}_{\mathrm{p}}+\mathrm{ka}_{\mathrm{p}}\right)+\mathrm{b}_{\mathrm{w}} \mathrm{s}_{\mathrm{p}} \mathrm{w}}{\mathrm{w}_{\mathrm{p}}\left(1-\delta_{\mathrm{p}}\right)+\delta_{\mathrm{p}} \mathrm{w}_{\mathrm{p}}^{\prime}+\mathrm{m}_{\mathrm{p}}}$.

For $\ddot{\mathrm{i}}_{\mathrm{p}}=0$, we obtain $\tilde{\mathrm{i}}_{\mathrm{p}}=\frac{\left(1-\delta_{\mathrm{p}}\right) \mathrm{w}_{\mathrm{p}} \mathrm{e}_{\mathrm{p}}}{\Upsilon_{\mathrm{p}}^{\prime}+\mathrm{m}_{\mathrm{p}}}$.

For $\dot{\mathrm{a}}_{\mathrm{p}}=0$, we obtain $\tilde{\mathrm{a}}_{\mathrm{p}}=\frac{\delta_{\mathrm{p}} \mathrm{w}_{\mathrm{p}}^{\prime} \mathrm{e}_{\mathrm{p}}}{\Upsilon_{\mathrm{p}}^{\prime}+\mathrm{m}_{\mathrm{p}}}$.

For $\dot{\mathrm{r}}_{\mathrm{p}}=0$, we obtain $\tilde{r}_{\mathrm{p}}=\frac{\Upsilon_{\mathrm{p}} i_{p}+\Upsilon_{\mathrm{p}}^{\prime} a_{p}}{\mathrm{~m}_{\mathrm{p}}}$.

For $\dot{w}=0$, we obtain $\tilde{w}=\mathrm{i}_{\mathrm{p}}+\mathrm{ca}_{\mathrm{p}}$. 
In order to achieve biologically relevant solutions, we find equilibrium points such that this system has positive solutions. There are two equilibrium points of system (11).

i) Disease free equilibrium point $E_{1}=\left(\widetilde{s_{p}}(t), 0,0,0,0,0\right)$. In this disease free equilibrium point, the population of five compartments of categorized people tends to zero and the system can be recognized in healthy situation.

ii) Endemic equilibrium point $E_{2}=\left(\widetilde{s_{p}}(t), \widetilde{e_{p}}(t), \widetilde{i_{p}}(t), \widetilde{a_{p}}(t), \widetilde{r_{p}}(t), \tilde{w}(t)\right)$ where its components are expressed above.

\section{Numerical Simulation}

In this section, we get deep insights into the model's dynamical behavior. For numerical simulation, the results of the implementation are given by the known Euler-Maruyama method [42]. We consider the following discretization model for $t=0, \Delta t, 2 \Delta t, \ldots, n \Delta$ and $k=1,2, \ldots, n$.

$$
\begin{aligned}
\dot{s}_{p_{k+1}}= & \left(n_{p}-m_{p} s_{p_{k}}-s_{p_{k}} b_{p}\left(i_{p}+k a_{p}\right)-s_{p_{k}} w b_{w}\right) \Delta t \\
& +\sigma_{1} \delta B_{1_{k}}-S_{p_{k}} \sigma_{2} \delta B_{2_{k}}-S_{p_{k}}\left(i_{p}+k a_{p}\right) \sigma_{3} \delta B_{3_{k}}-S_{p_{k}} w \sigma_{4} \delta B_{4_{k}}, \\
\dot{e}_{p_{k+1}}= & \left(S_{p_{k}} b_{p}\left(i_{p}+k a_{p}\right)+b_{w} s_{p_{k}} w-w_{p} e_{p_{k}}+\delta_{p} w_{p} e_{p_{k}}-\delta_{p} w_{p}^{\prime} e_{p_{k}}-m_{p} e_{p_{k}}\right) \Delta t \\
& +s_{p_{k}} \sigma_{3}\left(i_{p}+k a_{p}\right) \Delta B_{3_{k}}+s_{p_{k}} w \sigma_{4} \Delta B_{4_{k}}+w_{p} e_{p} \sigma_{5} \Delta B_{5_{k}}-w_{p}^{\prime} e_{p} \sigma_{5} \Delta B_{5_{k}}-e_{p} \sigma_{2} \Delta B_{2_{k}}, \\
\dot{i}_{p_{k+1}}= & \left(w_{p} e_{p}-\delta_{p} w_{p} e_{p}-\gamma_{p} i_{p_{k}}-i_{p_{k}} m_{p}\right) \Delta t-i_{p_{k}} \sigma_{2} \Delta B_{2 k}-\sigma_{5} w_{p} e_{p} \Delta B_{5 k}, \\
\dot{a}_{p_{k+1}}= & \left(\delta_{p} w_{p}^{\prime} e_{p}-\gamma_{p}^{\prime} a_{p_{k}}-m_{p} a_{p_{k}}\right) \Delta t-a_{p_{k}} \sigma_{2} \Delta B_{2 k}, \\
\dot{r}_{p_{k+1}}= & \left(\gamma_{p} i_{p}+\gamma_{p}^{\prime} a_{p}-r_{p_{k}} m_{p}\right) \Delta t-r_{p_{k}} \sigma_{2} \Delta B_{2 k}, \\
\dot{w}_{k+1}= & \epsilon\left(i_{p}+c a_{p}-w_{k}\right) \Delta t+\sigma_{6}\left(i_{p}+c a_{p}-w_{k}\right) \Delta B_{6 k},
\end{aligned}
$$

where $\Delta B_{i}(t) \sim \sqrt{\Delta t} Z_{i}$ and $Z_{i} \sim \mathcal{N}(0,1), i=1,2, \ldots, 6$. The source code has been written in MATLAB R2017b. Data given in Tab. 2 were calibrated for the cases in China, the first big source of COVID-19. On 21 February 2020 China (and WHO) informed that they have revised their guidance on case classification for COVID-19, removing the classification of clinically diagnosed previously used for Hubei province, and retaining only suspected and confirmed for all areas, the latter requiring laboratory confirmation. Some previously reported clinically diagnosed cases are thus expected to be discarded over the coming days as laboratory testing is conducted and some are found to be COVID19-negative (see [43]). Thus, in order to use more accurate and reliable data, we use the reported data in China before October 2020.

Table 2: The parameters values used in Example 5.1

\begin{tabular}{llllllllllll}
\hline$n_{p}$ & $m_{p}$ & $\epsilon$ & $k$ & $c$ & $b_{p}$ & $b_{w}$ & $w_{p}$ & $w_{p}^{\prime}$ & $\delta_{p}$ & $\gamma_{p}$ & $\gamma$ \\
\hline 0.0018 & 0.0018 & 0.1 & 0.5 & 0.5 & 0.01 & 0.5 & 0.1923 & 0.1923 & 0.65 & 0.1724 & 0.1724 \\
\hline
\end{tabular}

Example 5.1 In order to illustrate the adaptation and compatibility of the presented model with the deterministic model, we used the reported data in Wuhan City, China [21]. The parameters values used in the numerical simulation are given in Tab. 2 . 
The world health organization (WHO) reported an incubation period for COVID-19 between 2-10 days and the United States center for Disease Prevention and Control (CDC) estimates the incubation period for COVID-19 to be between 2-14 days. The mean incubation was 5.2 days (95\% confidence interval CI: 4.1 to 7.0). According to the report of WHO on 3 March 2020, globally about $3.4 \%$ of reported COVID-19 cases have died. In this simulation, we assume that $w_{p}=w_{p}^{\prime}$ and we set the infectious period of the cases as 5.8 days [21]. Therefore, we set $\gamma_{p}=0.1724$. Since the Coronavirus can live for hours to days on surfaces, so we set $\epsilon=0.1$ for 10 days lifetime of virus. Meanwhile, the transmission rates in our model depend on the epidemiological status and environmental conditions which change with time. The other parameters values used in this simulation are assumed based on the reported data in Wuhan City [21]. Also, initial conditions have been chosen as $\left(s_{p}(0), e_{p}(0), i_{p}(0), a_{p}(0), r_{p}(0), w(0)\right)=(0.1,0.1,0.1,0.1,0.1,0.1)$ and $\sigma_{i}=0.4, i=1,2, \ldots, 6$. The strength of the presented numerical method is particularly demonstrated in Fig. 2 which shows the adaptation of our model with the deterministic model.

In this example, if the intensities of the white noises of model (2) increase, the solution of model (2) will fluctuate around the disease free equilibrium. Fig. 3 shows the simulations with $\sigma_{i}=0.6, i=$ $1,2, \ldots, 6$.
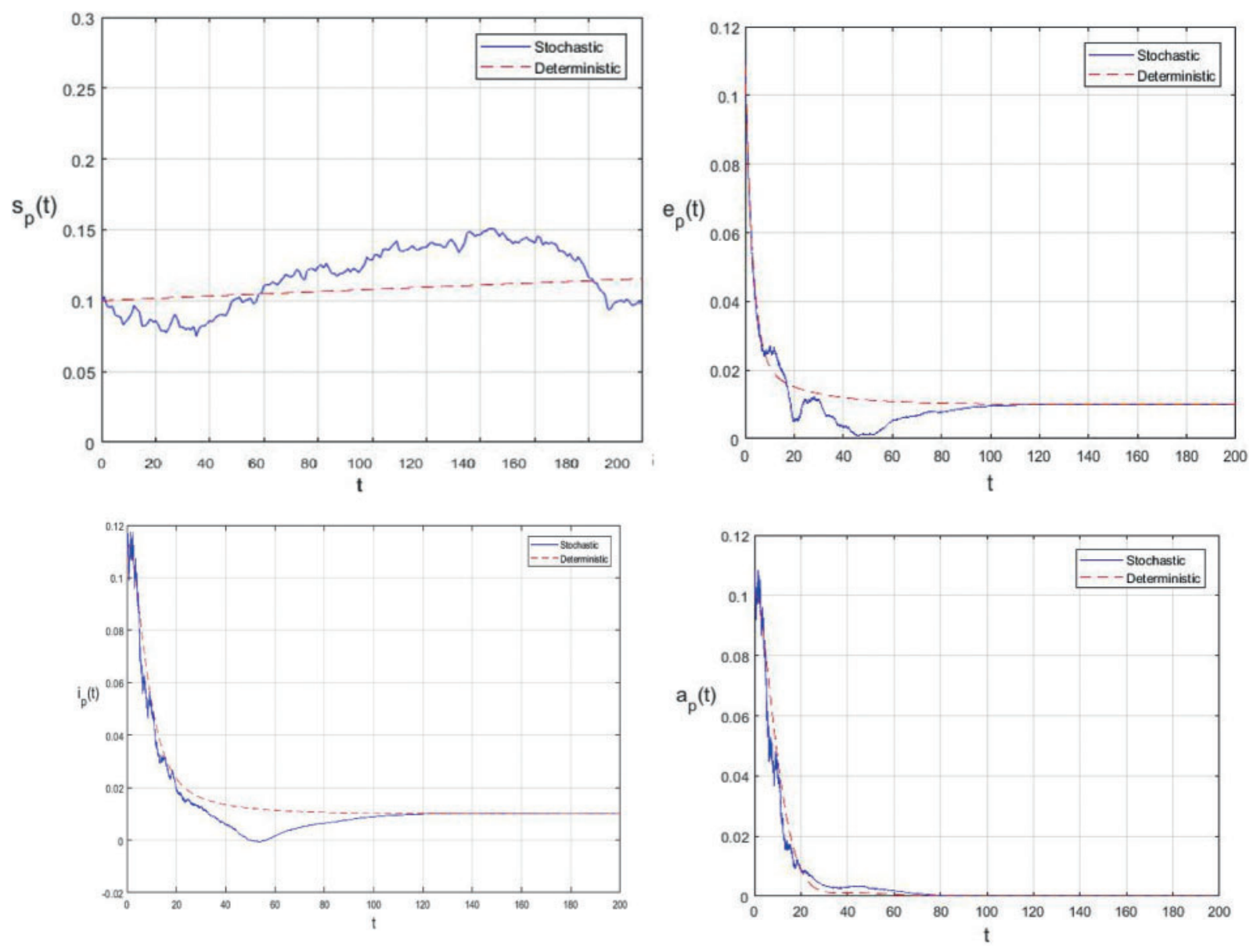

Figure 2: (Continued) 

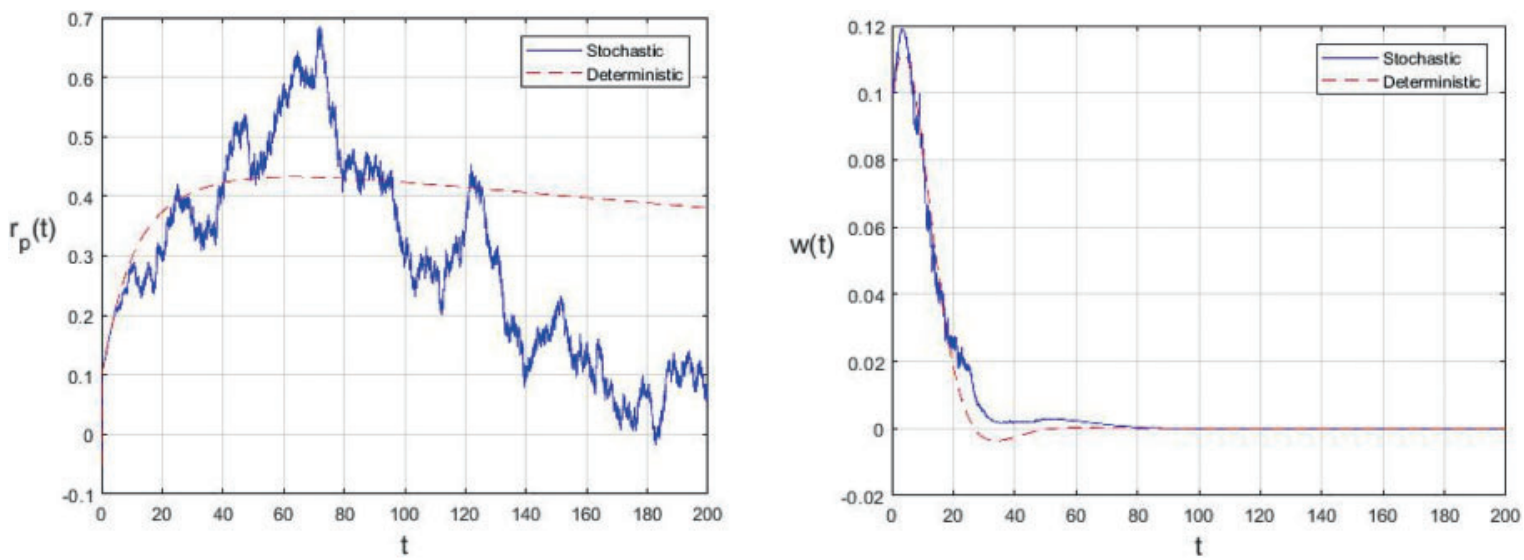

Figure 2: Comparison between the our presented stochastic model and deterministic model $\left(\sigma_{i}=0.4\right)$
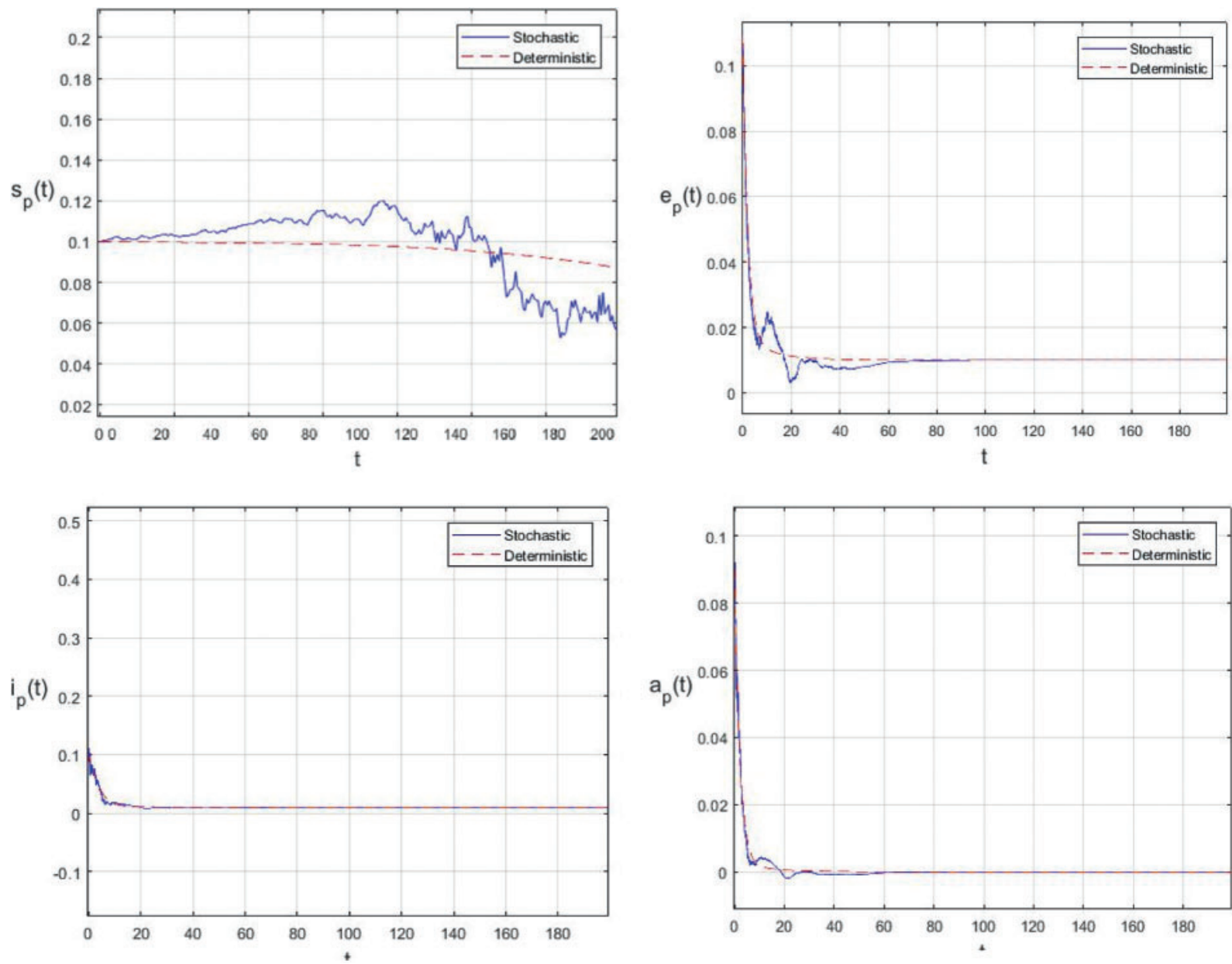

Figure 3: (Continued) 

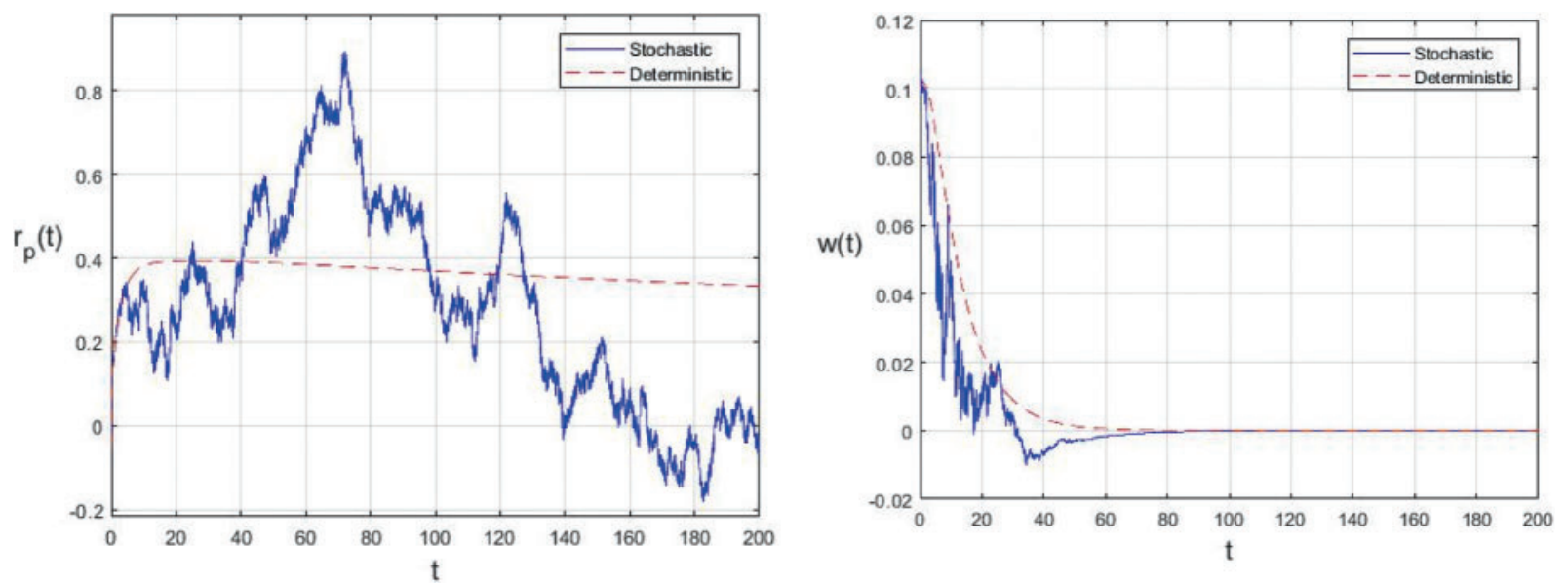

Figure 3: Comparison between the our presented stochastic model and deterministic model $\left(\sigma_{i}=0.6\right)$

Table 3: The parameters values used in Example 5.2

\begin{tabular}{llllllllllll}
\hline$n_{p}$ & $m_{p}$ & $\epsilon$ & $k$ & $c$ & $b_{p}$ & $b_{w}$ & $w_{p}$ & $w_{p}^{\prime}$ & $\delta_{p}$ & $\gamma_{p}$ & $\gamma$ \\
\hline 0.0018 & 0.0018 & 0.1 & 0.5 & 0.5 & 0.01 & 0.5 & 0.1923 & 0.1923 & 0.65 & 0.080 & 0.080 \\
\hline
\end{tabular}

Example 5.2 Since the information is changing and due to the lack of complete data on many parameters used in the proposed model, we now present some numerical simulations to illustrate the efficiency of our approach as an experiment for considering sensitivity analysis. Sensitivity analysis is commonly used to determine the robustness of model predictions to parameter values, since there are usually errors in collected data and assumptive parameter values. The parameter values used in this example are given in Tab. 3. In this example, we decrease the value of $\gamma$ and $\gamma_{p}$, so the infectious period of symptomatic and asymptomatic infection of people will increase. Fig. 4 shows that the time interval for occurring extinction of the disease increases when the infectious period of symptomatic and asymptomatic infection of people increases.

Example 5.3 In this example we take the intensities of the white noises of the model (2) as $\sigma_{2}=0.7$, $\sigma_{5}=0.8$ and $\sigma_{1}=\sigma_{3}=\sigma_{4}=\sigma_{6}=0.4$ which satisfy the condition of Theorem 4.3. The other parameter values used in this example are given in Tab. 4. On the basis of simulation, Fig. 5 shows that the number of symptomatic infected people $i_{p}$ will decay to zero under suitable conditions presented in Theorem 4.3. Therefore numerical simulation confirms the effect of high intensity white noises on the prevalence or extinction of the COVID-19. 

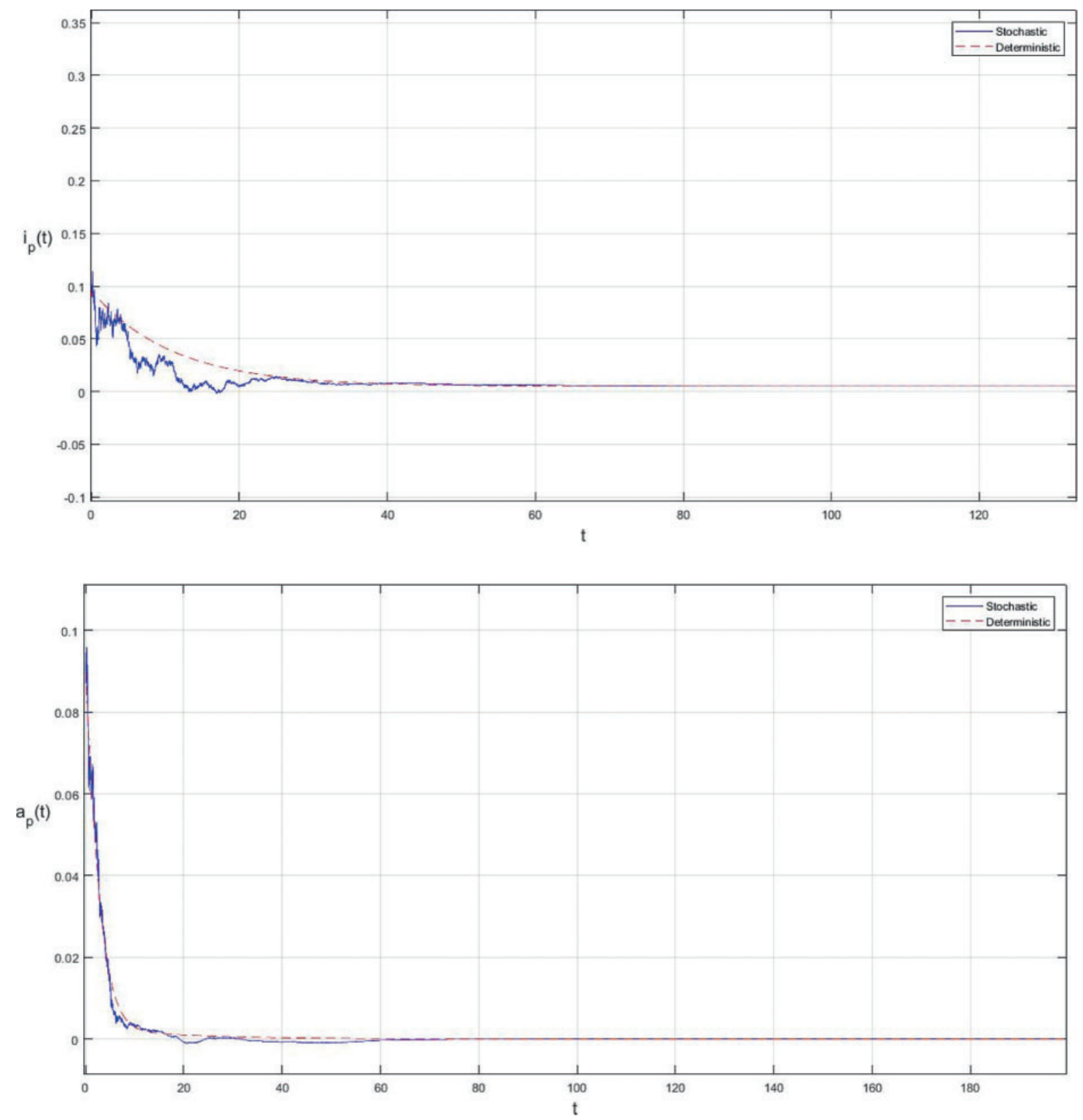

Figure 4: Effect of increasing the infectious period of symptomatic and asymptomatic infection of people

Table 4: The parameters values used in Example 5.3

\begin{tabular}{llllllllllll}
\hline$n_{p}$ & $m_{p}$ & $\epsilon$ & $k$ & $c$ & $b_{p}$ & $b_{w}$ & $w_{p}$ & $w_{p}^{\prime}$ & $\delta_{p}$ & $\gamma_{p}$ & $\gamma$ \\
\hline 0.0018 & 0.0018 & 0.1 & 0.5 & 0.5 & 0.01 & 0.6 & 0.1923 & 0.1923 & 1.1 & 0.650 & 0.1724 \\
\hline
\end{tabular}




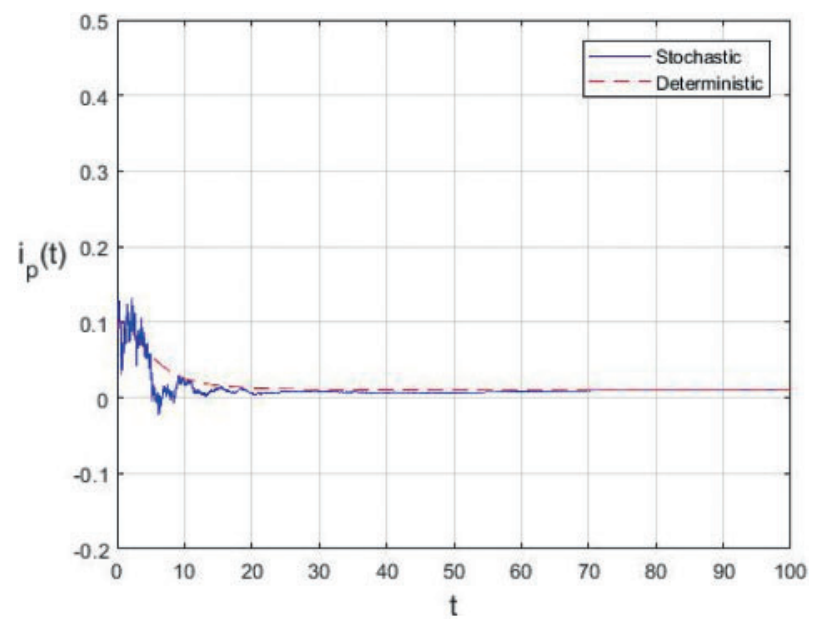

Figure 5: Extinction of the stochastic model under suitable conditions

\section{Results and Discussion}

In the proposed approach, the results have been obtained using the known Euler-Maruyama method as mentioned above. Moreover, to ensure the accuracy and precision of the obtained results, the comparison of the proposed scheme has been done with the deterministic model. In stochastic models, there are random variations due to uncertainties in the parameter. According to the presented comparisons (see Fig. 2 and Fig. 3), the results of our stochastic epidemic model are convergent to the deterministic model. The simulation results demonstrate the efficiency of our model, and the possibility of comparability of the stochastic model with the deterministic model. Evaluation results and dynamical analysis confirmed its effectiveness, efficiency, and user satisfaction. In this paper, we found that the infection can be controlled by suitable health decisions and herd immunity as a naturally occurring phenomenon can make the number of infectious cases descending. Furthermore, numerical simulation confirms the effect of high intensity white noises on the prevalence or extinction of the COVID-19. From the simulated findings (please see Fig. 4 and Fig. 5), it can be noticed that the our stochastic model shows a strong agreement with the deterministic case at different values of white noise. Additionally, graphical investigations confirmed that the infectious population tends to zero under suitable extinction conditions presented in Theorem 4.3. When the noises $\sigma_{2}$ and $\sigma_{5}$ are sufficiently large then the disease goes extinct. The white noises $\sigma_{2}$ and $\sigma_{5}$ are related to the fluctuation of $m_{p}$ (the death rate of people) and $\delta_{p}$ (the proportion of asymtomatic infection of people), respectively.

\section{Conclusion and Remarks}

In this paper, we have developed and analyzed an epidemic model for simulating transmissibility of the COVID-19 based on Reservoir-People transmission network. We construct a stochastic version of the Reservoir-People model by using the white noise and Brownian motion. Also, we proved that there is a unique solution for the presented stochastic model. Moreover, the equilibria of the system are considered. Also, we establish the extinction of the disease under some suitable conditions. Finally, the numerical simulation is carried out to demonstrate the efficiency of our model, and the possibility of comparability of the stochastic model with the deterministic model. Furthermore, numerical results demonstrate the validity of suitable conditions for the extinction of the disease. Since mathematical 
models are powerful tools to understand the dynamics of real world phenomena, particularly the transmission of an infectious disease, therefore this paper could lead to other studies that have included random and uncertainty of the model and can be more consistent with the reality of Coronavirus transmission. The presented results may be fruitful for the existing outbreak in a better way and can be used in taking defensive techniques to decrease the infection. The area of stochastic modeling has been extended recently, therefore this model is an indication for further study in this area. After getting high motivation from this paper and using ideas of modeling approaches in new researches (see [43-52]), hybrid stochastic fractional approach will be considered to investigate the dynamics of the COVID-19, as a scope of future works.

Funding Statement: The authors received no specific funding for this study.

Conflicts of Interest: The authors declare that they have no conflicts of interest to report regarding the present study.

\section{References}

[1] H. W. Hethcote, "The mathematics of infectious diseases," SIAM Review, vol. 42, no. 4, pp. 599-653, 2000.

[2] N. Shahid, D. Baleanu, N. Ahmed, T. S. Shaikh, A. Raza et al., "Optimality of solution with numerical investigation for coronavirus epidemic model," Computers Materials \& Continua, vol. 67, no. 2, pp. 17131728, 2021.

[3] D. Kramer, "Covid-19 pandemic modeling is fraught with uncertainties," Physics Today, vol. 73, no. 6, pp. 25-27, 2020.

[4] J. X. Yang, "The spreading of infectious diseases with recurrent mobility of community population," Physica A: Statistical Mechanics and its Applications, vol. 541, Article ID 123316, 2020.

[5] T. A. Yildiz and E. Karaoğlu, "Optimal control strategies for tuberculosis dynamics with exogenous reinfections in case of treatment at home and treatment in hospital," Nonlinear Dynamics, vol. 97, no. 4, pp. 2643-2659, 2019.

[6] M. S. Arif, A. Raza, M. Rafiq and M. Bibi, "A reliable numerical analysis for stochastic hepatitis B virus epidemic model with the migration effect," Iranian Journal of Science and Technology, Transactions A, vol. 43, no. 5, pp. 2477-2492, 2019.

[7] J. E. Macías-Díaz, N. Ahmed and M. Rafiq, "Analysis and nonstandard numerical design of a discrete three-dimensional hepatitis B epidemic model," Mathematics, vol. 7, no. 12, Article ID 1157, 2019.

[8] D. Baleanu, S. Sajjadi, A. Jajarmi, O. Defterli and J. H. Asad, "The fractional dynamics of a linear triatomic molecule," Romanian Reports in Physics, vol. 73, no. 1, Article ID 105, 2021.

[9] N. Ahmed, M. Jawaz, M. Rafiq, M. A. Rehman, M. Ali et al., "Numerical treatment of an epidemic model with spatial diffusion," Journal of Applied Environmental and Biological Sciences, vol. 8, no. 6, pp. 17-29, 2018.

[10] J. E. Macias-Diaz, A. Raza, N. Ahmed and M. Rafiq, "Analysis of a nonstandard computer method to simulate a nonlinear stochastic epidemiological model of coronavirus-like diseases," Computer Methods and Programs in Biomedicine, vol. 204, Article ID 106054, 2021.

[11] A. Raza, A. Ahmadian, M. Rafiq, S. Salahshour and M. Ferrara, "An analysis of a nonlinear susceptibleexposed-infected-quarantine-recovered pandemic model of a novel coronavirus with delay effect," Results in Physics, vol. 21, Article ID 103771, 2021.

[12] T. Sun and Y. Wang, "Modeling COVID-19 epidemic in heilongjiang province, China," Chaos, Solitons \& Fractals, vol. 138, Article ID 109949, 2020.

[13] P. Melin, J. C. Monica, D. Sanchez and O. Castillo, "Analysis of spatial spread relationships of coronavirus (COVID-19) pandemic in the world using self-organizing maps," Chaos, Solitons \& Fractals, vol. 138, Article ID 109917, 2020. 
[14] A. Yousefpour, H. Jahanshahi and S. Bekiros, "Optimal policies for control of the novel coronavirus disease (COVID-19) outbreak," Chaos, Solitons \& Fractals, vol. 136, Article ID 109883, 2020.

[15] B. Tang, X. Wang, Q. Li, N. L. Bragazzi, S. Tang et al., "Estimation of the transmission risk of the 2019nCov and its implication for public health interventions," Journal of Clinical Medicine, vol. 9, no. 2, Article ID 462, 2020.

[16] P. Wu, X. Hao, E. H. Y. Lau, J. Y. Wong, K. S. M. Leung et al., "Real-time tentative assessment of the epidemiological characteristics of novel coronavirus infections in wuhan, China, as at 22 January 2020," Eurosurveillance, vol. 25, no. 3, Article ID 2000044, 2020.

[17] T. Sun and Y. Wang, "Modeling covid-19 epidemic in heilongiiang province, China," Chaos, Solitons \& Fractals, vol. 138, Article ID 109949, 2020.

[18] D. Dansana, R. Kumar, A. Parida, R. Sharma, J. D. Adhikari et al., "Using susceptible-exposed-infectiousrecovered model to forecast coronavirus outbreak," Computers Materials \& Continua, vol. 67, no. 2, pp. $1595-1612,2021$.

[19] M. Naveed, M. Rafiq, A. Raza, N. Ahmed, I. Khan et al., "Mathematical analysis of novel coronavirus (2019-ncov) delay pandemic model," Computers, Materials \& Continua, vol. 64, no. 3, pp. 1401-1414, 2020.

[20] M. Tahir, T. Shah, G. Zaman and T. Khan, "Stability behavior of mathematical model mERS corona virus spread in population," Filomat, vol. 33, no. 12, pp. 3947-3960, 2019.

[21] T. M. Chen, J. Rui, Q. P. Wang, Z. Y. Zhao, J. A. Cui et al., "A mathematical model for simulating the phase-based transmissibility of a novel coronavirus," Infectious Diseases of Poverty, vol. 9, no. 1, pp. 1-8, 2020.

[22] E. Hashemizadeh and M. Ebadi, "A numerical solution by alternative legendre polynomials on a model for novel coronavirus (covid-19)," Advances in Difference Equations, vol. 202, Article ID 527, 2020.

[23] W. Gao, H. M. Baskonus and L. Shi, "New investigation of bats-hosts-reservoir-people coronavirus model and application to 2019-ncov system," Advances in Difference Equations, vol. 2020, Article ID 391, 2020.

[24] S. Cai, Y. Cai and X. Mao, "A stochastic differential equation sIS epidemic model with two correlated brownian motions," Nonlinear Dynamics, vol. 97, pp. 2175-2187, 2019.

[25] M. Fahimi, K. Nouri and L. Torkzadeh, "Chaos in a stochastic cancer model," Physica A: Statistical Mechanics and its Applications, vol. 545, Article ID 123810, 2020.

[26] H. Mehrjerdi, R. Hemmati and E. Farrokhi, "Nonlinear stochastic modeling for optimal dispatch of distributed energy resources in active distribution grids including reactive power," Simulation Modelling Practice and Theory, vol. 94, pp. 1-13, 2019.

[27] W. Shatanawi, A. Raza, M. S. Arif, K. Abodayeh, M. Rafiq et al., "An effective numerical method for the solution of a stochastic coronavirus (2019-ncovid) pandemic model," Computers, Materials \& Continua, vol. 66, no. 2, pp. 1121-1137, 2021.

[28] W. Shatanawi, A. Raza, M. S. Arif, K. Abodayeh, M. Rafiq et al., "Design of nonstandard computational method for stochastic susceptible-infected-treated-recovered dynamics of coronavirus model," Advances in Difference Equations, vol. 2020, Article ID 505, 2020.

[29] J. Danane, K. Allali, Z. Hammouch and K. S. Nisar, "Mathematical analysis and simulation of a stochastic cOVID-19 levy jump model with isolation strategy," Results in Physics, vol. 23, Article ID 103994, 2021.

[30] K. Abodayeh, A. Raza, M. S. Arif, M. Rafiq, M. Bibi et al., "Stochastic numerical analysis for impact of heavy alcohol consumption on transmission dynamics of gonorrhoea epidemic," Computers, Materials \& Continua, vol. 62, no. 3, pp. 1125-1142, 2020.

[31] A. Raza, M. Rafiq, D. Baleanu and M. S. Arif, "Numerical simulations for stochastic meme epidemic model," Advances in Difference Equations, vol. 2020, Article ID 176, 2020.

[32] W. Shatanawi, S. Arif, A. Raza, M. Rafiq, M. Bibi et al., "Structure-preserving dynamics of stochastic epidemic model with the saturated incidence rate," Computers Materials \& Continua, vol. 64, no. 2, pp. 797-811, 2020.

[33] A. Raza, M. Rafiq, N. Ahmed, I. Khan, K. S. Nisar et al., "A structure preserving numerical method for solution of stochastic epidemic model of smoking dynamics," Computers, Materials \& Continua, vol. 65, no. 1, pp. 263-278, 2020. 
[34] M. A. Noor, A. Raza, M. S. Arif, M. Rafiq, K. S. Nisar et al., "Nonstandard computational analysis of the stochastic cOVID-19 pandemic model: An application of computational biology," Alexandria Engineering Journal, vol. 61, no. 1, pp. 619-630, 2021.

[35] K. Chatterjee, K. Chatterjee, A. Kumar and S. Shankar, "Healthcare impact of COVID-19 epidemic in India: A stochastic mathematical model," Medical Journal Armed Forces India, vol. 76, pp. 147-155, 2020.

[36] S. He, S. Tang and L. Rong, "A discrete stochastic model of the COVID-19 outbreak: Forecast and control," Mathematical Biosciences and Engineering, vol. 17, pp. 2792-2804, 2020.

[37] T., Rediat, "Stochastic modelling for predicting covid-19 prevalence in east Africa countries," Infectious Disease Modelling, vol. 5, pp. 598-607, 2020.

[38] X. Mao, Stochastic Differential Equations and Applications, Horwood Publishing, Chichester, 1997.

[39] S. Cobzas, R. Miculescu and A. Nicolae, Stochastic Differential Equations and Applications, Springer International Publishing, Switzerland, 2019.

[40] E. Pap, Handbook of Measure Theory, Amsterdam, North-Holland, 2002.

[41] D. J. Higham, "An algorithmic introduction to numerical simulation of stochastic differential equations," SIAM Review, vol. 43, no. 3, pp. 525-546, 2001.

[42] B. Ivorra, M. R. Ferrández, M. Vela-Pérez and A. M. Ramos, "Mathematical modeling of the spread of the coronavirus disease 2019 (covid-19) taking into account the undetected infections. the case of China," Communications in Nonlinear Science and Numerical Simulation, vol. 88, Article ID 105303, 2020.

[43] D. Baleanu, S. Zibaei, M. Namjoo and A. Jajarmi, "A nonstandard finite difference scheme for the modeling and nonidentical synchronization of a novel fractional chaotic system," Advances in Difference Equations, vol. 2021, Article ID 308, 2021.

[44] D. Baleanu, S. Sajjadi, J. H. Asad, A. Jajarmi and E. Stiri, "Hyperchaotic behaviors, optimal control, and synchronization of a nonautonomous cardiac conduction system," Advances in Difference Equations, vol. 2021, Article ID 157, 2021.

[45] D. Baleanu, S. Sajjadi., A. Jajarmi and O. Defterli, "On a nonlinear dynamical system with both chaotic and nonchaotic behaviors: a new fractional analysis and control," Advances in Difference Equations, vol. 2021, Article ID 234, 2021.

[46] A. Jajarmi, D. Baleanu, K. Zarghami, K. Zarghami and S. Mobayen, "A general fractional formulation and tracking control for immunogenic tumor dynamics," Mathematical Methods in the Applied Sciences, Inpress.

[47] M. Fiza, A. Alsubie, H. Ullah, N. H. Hamadneh, S. Islam et al., "Three-dimensional rotating flow of MHD jeffrey fluid flow between two parallel plates with impact of hall current," Mathematical Problems in Engineering, vol. 2021, Article ID 6626411, 2021.

[48] H. Ullah, A. Alsubie, M. Fiza, N. H. Hamadneh, S. Islam et al., "Impact of hall current and nonlinear thermal radiation on jeffrey nanofluid flow in rotating frame," Mathematical Problems in Engineering, vol. 2021, Article ID 9930017, 2021.

[49] I. Khan, H. Ullah, M. Fiza, S. Islam, A. Z. Raja et al., "A levenberg-marquardt backpropagation method for unsteady squeezing flow of heat and mass transfer behaviour between parallel plates," Advances in Mechanical Engineering, vol. 13, pp. 1-15, 2021.

[50] A. Yazidi and H. L. Hammer, "Solving stochastic nonlinear resource allocation problems using continuous learning automata," Applied Intelligence, vol. 48, no. 11, pp. 4392-4411, 2018.

[51] H. Ullah, I. Khan, I. Khan, N. H. Hamadneh, M. F. Al-Asad et al., "Mhd boundary layer flow over a stretching sheet: A new stochastic method," Mathematical Problems in Engineering, vol. 2021, Article ID 9924593, 2021.

[52] I. Khan, H. Ullah, H. Alsalman, M. Fiza, S. Islam et al., "Falkner-Skan equation with heat transfer: A new stochastic numerical approach," Mathematical Problems in Engineering, vol. 2021, Article ID 3921481, 2021. 